\title{
Group I metabotropic glutamate receptor mediated dynamic immune dysfunction in children with fragile $\mathrm{X}$ syndrome
}

Milo Careaga ${ }^{1,2}$, Tamanna Noyon ${ }^{2}$, Kirin Basuta ${ }^{3}$, Judy Van de Water ${ }^{2,4}$, Flora Tassone ${ }^{2,3}$, Randi J Hagerman ${ }^{2,5}$ and Paul Ashwood ${ }^{1,2^{*}}$

\begin{abstract}
Background: Fragile $X$ syndrome (FXS) is the leading cause of inheritable intellectual disability in male children, and is predominantly caused by a single gene mutation resulting in expanded trinucleotide CGG-repeats within the $5^{\prime}$ untranslated region of the fragile X mental retardation (FMR1) gene. Reports have suggested the presence of immune dysregulation in FXS with evidence of altered plasma cytokine levels; however, no studies have directly assessed functional cellular immune responses in children with FXS. In order to ascertain if immune dysregulation is present in children with FXS, dynamic cellular responses to immune stimulation were examined.

Methods: Peripheral blood mononuclear cells (PBMC) were from male children with FXS $(n=27)$ and from male aged-matched typically developing (TD) controls $(n=8)$. PBMC were cultured for 48 hours in media alone or with lipopolysaccharides (LPS; $1 \mu \mathrm{g} / \mathrm{mL}$ ) to stimulate the innate immune response or with phytohemagglutinin (PHA; $8 \mu \mathrm{g} / \mathrm{mL}$ ) to stimulate the adaptive T-cell response. Additionally, the group I mGluR agonist, DHPG, was added to cultures to ascertain the role of mGluR signaling in the immune response in subject with FXS. Supernatants were harvested and cytokine levels were assessed using Luminex multiplexing technology.

Results: Children with FXS displayed similar innate immune response following challenge with LPS alone when compared with TD controls; however, when LPS was added in the presence of a group I mGluR agonist, DHPG, increased immune response were observed in children with FXS for a number of pro-inflammatory cytokines including IL-6 $(P=0.02)$, and IL-12p40 $(P<0.01)$. Following PHA stimulation, with or without DHPG, no significant differences between subjects with FXS and TD were seen.

Conclusions: In unstimulated cultures, subjects with FXS did not display altered dynamic immune response to LPS or PHA alone; however, subjects with FXS showed an altered response to co-current stimulation of LPS and DHPG, such that subjects with FXS failed to inhibit production of pro-inflammatory cytokines, suggesting a role of group I mGluR signaling in innate immune responses in FXS.
\end{abstract}

Keywords: Fragile x syndrome, Immune, Cytokine, Metabotropic glutamate receptor

\footnotetext{
* Correspondence: pashwood@ucdavis.edu

'Department of Medical Microbiology and Immunology, and the MIND Institute, UC Davis, 2805, 50th Street, Sacramento, CA 95817, USA ${ }^{2}$ MIND Institute, UC Davis Medical Center, Sacramento, CA, USA

Full list of author information is available at the end of the article
} 


\section{Introduction}

Fragile X syndrome (FXS) is a single-gene disorder nearly always caused by an unstable mutation in the fragile X mental retardation 1 (FMR1) X-linked gene and results from the expansion of a trinucleotide (CGG) repeat sequence in the 5'UTR of this gene [1]. The full mutation, present in individuals having an FMR1 allele with more than 200 CGG repeats, typically is methylated with partial or complete transcriptional silencing of the FMR1 gene, leading to a reduction or absence of the FMR1 protein, FMRP [1-4]. Although FXS is associated with a characteristic phenotype, there is considerable within-syndrome variation in the severity of affectedness and the profile of impairments including a significant (approximately 30\%) comorbidity with autism spectrum disorders (ASD) $[5,6]$.

Recent evidence suggests that there is immune dysregulation in individuals with FXS that may play a role in the disease process [7]. Neuroimmune interactions begin during early neurodevelopment and continue throughout life, with the immune system supporting many aspects of neural function. Alterations in the immune system would increase sensitivity to neurologic damage from a variety of sources including infection and exposure to xenobiotics, particularly during early development. Indeed, an increased frequency of infections has been reported in a subgroup of boys with FXS, especially in early childhood [8]. This may be the result of an aberrant or dysfunctional immune response in this group, and possibly contributes to the frequently observed severe cognitive deficit and language impairment [8]. Consequently, there is a need for research that can identify the biological basis of the immune anomalies in FXS, which may facilitate future treatments.

Groundbreaking advances in the FXS field have paved the way for treatment of the underlying neurobiology of the disorder. The 'mGluR theory' of FXS, which is strongly supported by several lines of evidence, states that mental impairment and phenotypic behaviors associated with FXS arise, at least in part, from constitutive activation of translational pathways normally controlled by group 1 metabotropic glutamate receptor (mGluR1 and mGluR5) activity [9]. Group I mGluRs are involved in numerous functions including learning and memory, and are expressed in both the central and peripheral nervous system [10]. In neurons, mGluRs can augment synapse excitability and thereby alter the function of other receptors including GABA receptors [11].

mGluRs are expressed on peripheral blood mononuclear cells (PBMC), where they are believed to be involved in immune development, activation, response, and survival [12-15]. mGluRs are members of the group $C$ family of G-protein-coupled receptors, and function by signaling through $G$ proteins to activate secondary messengers.
They are responsive to low concentrations of glutamate, such as that observed in the periphery, where glutamate levels are typically between 20 to $40 \mu \mathrm{M}$ in plasma [16]; considerably lower than what is found in synaptic clefs within the central nervous system (CNS) where glutamate levels can reach millimolar levels [17]. Group I mGluRs in particular, are expressed differentially in naïve and activated T-cells, with mGluR5 being constitutively expressed and mGluR1 only being expressed in activated T-cells [18]. In addition, mGluRs on T-cells have been shown to be differentially coupled to intracellular signaling systems such as cAMP, and ERK pathways [15]. It is thought that the differential expression on the cell surface and coupling to signaling mechanisms contributes to T-cell activation, expression of pro-inflammatory cytokines, and cell survival. In addition, mGluRs are also differentially expressed on other immune cells such as microglia and macrophages and may be related to activation status [19].

The present study focused on how group I mGluR activation influences the immune response in pediatric populations of typically developing (TD) children compared to children with FXS, through an analysis of dynamic immune cell function. To date, no study has attempted to analyze the function of neurotransmitter receptors using PBMC in FXS. This study illustrates the role of mGluRs in immune cell function in both a neurotypical pediatric population as well as in children with FXS where mGluR signaling is altered, expanding upon previous immunological findings in FXS.

\section{Material and methods}

\section{Subjects}

Twenty-seven male subjects with FXS aged 2 to 9 years (median 5.4 years (interquartile range 3.7 to 7.5)) were recruited through the Fragile X Treatment and Research Center at the MIND Institute at University of California, Davis, CA, USA. The study also included 8 male TD controls aged 3 to 8 years (median 3.3 years (3.2 to 5.7 years)). Subjects on minocycline or other medications with established anti-inflammatory properties were excluded from the study. All participants with FXS underwent a clinical assessment that included a detailed medical history and medical examination, and measurements of typical genetic and physical features of FXS. In addition, subjects underwent IQ testing and adaptive skills testing using the following instruments: the Wechsler Scales of Intelligence [20] and the Vineland Adaptive Behavior Scales [21]. Controls were administered a medical examination and the Social Communication Questionnaire (SCQ) [22] to determine that they were typically developing, and none exceeded scores above 15 . In addition, a review of available prenatal, birth, and medical records were performed for all subjects. Clinical 
assessments were performed by trained clinicians under the supervision of one of the authors $(\mathrm{RJH})$, a pediatrician at the MIND Institute. The study protocol followed the ethical guidelines of the declaration of Helsinki and was approved by the Institutional Review Boards of the UC Davis School of Medicine and the State of California, and written informed consent was obtained from a legal guardian for all study subjects.

\section{CGG sizing}

Genomic DNA was isolated from 3 to $5 \mathrm{~mL}$ of peripheral blood leukocytes using standard procedures (Gentra Puregene kit; Qiagen, Valencia, CA). An initial centrifugation step on whole blood permitted plasma separation and storage before isolation of the DNA.

The size and methylation status of the CGG repeats was determined using both Southern blot and PCR analysis. Details of the Southern blot and PCR methods are in Tassone et al. [23] and Filipovic-Sadic et al. [24]. Analysis and calculation of the repeat size for both Southern blot and PCR analysis was carried out using an Alpha Innotech FluorChem 8800 Image Detection System (ProteinSimple, Santa Clara, CA)and the ABI 3730XL 96-Capillary Electrophoresis Genetic Analyzer (Life Technologies, Grand Island, NY).

\section{Cell isolation}

Peripheral blood was collected in an acid-citrate-dextrose Vacutainer tube (BD Biosciences; San Jose, CA, USA) and processed within 12 hours of collection. Blood was centrifuged for 10 minutes at 2,300 rpm, and plasma was collected and stored at $-80^{\circ} \mathrm{C}$. The remaining cells were mixed 1:1 with Hanks Balanced Salt Solution (HBSS; Gibco, Gaithersburg, MD, USA) without $\mathrm{Ca}^{2+}$ or $\mathrm{Mg}^{2+}$. The diluted blood was then carefully layered over a Ficoll-Paque gradient (Pharmacia Biotech, Piscataway, NJ, USA) and centrifuged at $1,700 \mathrm{rpm}$ for 30 minutes at room temperature. PBMC were then harvested from the interface layer and washed twice with HBSS. Viability was be determined by trypan blue exclusion. Cells were then resuspended at a concentration of $2 \times 10^{6}$ cells $/ \mathrm{mL}$ in tissue culture medium (TCM) consisting of: RPMI 1640 (Gibco, Gaithersburg, MD, USA) supplemented with 10\% low endotoxin, heat inactivated FBS (Omega Scientific; Tarzana, CA, USA), $100 \mathrm{IU} / \mathrm{mL}$ penicillin, and $100 \mathrm{IU} / \mathrm{mL}$ streptomycin (Sigma, St Louis, MO, USA).

\section{Cellular stimulations}

Isolated PBMC were stimulated for one hour in RPMI 1640 media with 10\% FBS (Gibco, Gaithersburg, MD, USA), $1 \%$ penicillin and streptomycin alone, or the addition of $100 \mu \mathrm{M}$ of a group I mGluR agonist (S)3,5-dihydroxyphenylglycine (DHPG) (DHPG; Tocris, Minneapolis, MN, USA), or $10 \mu \mathrm{M}$ of an mGluR5 specific antagonist 3-((2-methyl-1,3-thiazol-4-yl)ethynyl)pyridine hydrochloride (MTEP; Tocris, Minneapolis, MN, USA) followed by the addition of either $1.0 \mu \mathrm{g} / \mathrm{mL}$ lipopolysaccharide (LPS; Escherichia coli serotype 0111:B4, Sigma, St. Louis, MO, USA) or $8 \mu \mathrm{g} / \mathrm{mL}$ phytohemagglutinin (PHA; Sigma, St Louis, MO, USA) and cultured at $37^{\circ} \mathrm{C}$ with $5 \% \mathrm{CO}_{2}$ for another 47 hours (48 hours total). After this period, cells were collected and spun at 2,000 rpm for 10 minutes and supernatants were collected and stored at $-80^{\circ} \mathrm{C}$ until analyzed by Luminex multiplexing technology.

\section{Luminex multiplex analysis}

Quantification of IFN $\gamma$, granulocyte-macrophage colonystimulating factor (GM-CSF), IL-1 $\beta$, IL-6, IL-10, IL-12 (p40), IL-13, IL-17 and TNF $\alpha$ in the cell supernatants was determined using human multiplexing bead immunoassays (Millipore, Billerica, MA, USA). The cytokines GM-CSF, IL-1 $\beta$, IL-6, IL-10, TNF $\alpha$, and IL-12(p40) were used to evaluate innate immune responses after LPS stimulation and the cytokines GM-CSF, IFN $\gamma$, IL-10, IL-13, and IL-17 were used to assess responses after PHA stimulation. Samples were analyzed per manufacturer specifications. Specifically, $25 \mu \mathrm{L}$ of supernatant were incubated with antibody-coupled beads. After a series of washes, a biotinylated detection antibody was added to the beads, and the reaction mixture was detected by the addition of streptavidin-phycoerythrin. The bead sets were analyzed using a flow-based Luminex ${ }^{\text {Tx }} 100$ suspension array system (Bio-Plex 200; Bio-Rad Laboratories, Inc. Hercules, CA, USA). Unknown sample cytokine concentrations were calculated by Bio-Plex Manager software (Bio-Rad Laboratories, Inc. Hercules, CA, USA) using a standard curve derived from the known reference cytokine concentrations supplied by the manufacturer. A five-parameter model was used to calculate final concentrations and values are expressed in $\mathrm{pg} / \mathrm{mL}$. The sensitivity of this assay allowed the detection of cytokine concentrations with the following limits of detection: IFN $\gamma(0.4 \mathrm{pg} / \mathrm{mL})$, GM-CSF $(2.3 \mathrm{pg} / \mathrm{mL}), \mathrm{IL}-1 \beta$ (0.7 pg/mL), IL-6 (0.4 pg/mL), IL-10 (0.3 pg/mL), IL-12 (p40) $(12.3 \mathrm{pg} / \mathrm{mL}), \mathrm{IL}-13(0.3 \mathrm{pg} / \mathrm{mL}), \mathrm{IL}-17(0.4 \mathrm{pg} / \mathrm{mL})$, and TNF $\alpha(0.2 \mathrm{pg} / \mathrm{mL})$. Values below the limit of detection (LOD) were replaced with one half the LOD. Supernatant aliquots were free of any previous freeze/thaw cycle.

\section{Statistical analysis}

Data analysis was performed using STATA 12 software (College Station, TX, USA). Data was determined as non-parametric using Shapiro-Wilks test for normality. Wilcoxon matched-pairs signed-rank tests were used to compare cytokine levels within group pre and post stimulation and Wilcoxon-rank sum tests for between subject group comparisons. For comparison of relative 
immune response between group, outliers were determined if datum were greater than four median absolute deviations from the mean. A probability value $(P)$ of less than 0.05 was considered to be significant.

\section{Results}

PBMC from children with FXS and TD controls were stimulated with LPS, a Toll-like receptor (TLR)-4 agonist, for 48 hours to assess the dynamic response of their innate immune system. No significant differences were apparent in the supernatants collected from the cell cultures of TD controls compared with children with FXS in the cytokines assayed (GM-CSF, IL-1 $\beta$, IL-6, IL-10, TNF $\alpha$, and IL-12(p40)) (Table 1). When a group I mGluR agonist, DHPG, was added during stimulation there was a significant decrease in cytokines levels for GM-CSF $(P=0.04), \mathrm{IL}-12(\mathrm{p} 40)(P=0.01)$, and TNF $\alpha(P=0.03)$ in TD controls (Table 1$)$. In contrast, cell culture supernatants from children with FXS showed that select inflammatory cytokines levels were increased or remained unchanged after administration of DHPG to the culture $(P<0.01$; Table 1$)$. Further, the anti-inflammatory cytokine, IL-10, was decreased following stimulation in the presence of DHPG $(P<0.01$; Table 1$)$. The exception to these findings was IL- $1 \beta$ production, which was marginally increased in TD controls $(P=0.05)$ but still had a greater production in FXS children, with an over twofold increase $(P<0.01)$ when PBMC were stimulated with LPS plus DHPG (Table 1).

Overall, the apparent skewing in cytokine production could be observed as a differential response to LPS stimulation in the presence of DHPG compared with LPS alone (Figure 1A-D); such that TD controls displayed a significantly lower production of IL-6 (median $-17.3 \%$ (interquartile range -33.0 to $-0.4 \%)$ versus $11.4 \%(-11.7$ to $43.6 \%) ; P=0.02$ ) and IL-12(p40) (median $-48.7 \%$ (interquartile range -69.7 to $-19.8 \%)$ versus $0 \%(-21.1$ to $43.4 \%) ; P<0.01)$ in the presence of DHPG compared with children with FXS. In addition, both TNF $\alpha$ (median $-15.5 \%$ (interquartile range -30.8 to $-4.8 \%$ ) versus $-1.6 \%(-18.3$ to 25.8$) ; P=0.07)$ and $\mathrm{GM}-\mathrm{CSF}$ (median $-19.9 \%$ (interquartile range -28.0 to $-1.7 \%$ ) versus $6.7 \%(-23.5$ to $33.8 \%) ; P=0.11$ ) showed trends toward a higher differential response in TD controls compared children with FXS approaching statistical significance.

To determine if the basal level of glutamate in the culture media was altering baseline response of $\mathrm{PBMC}$ via a group I mGluR-dependent manner in children with FXS, a group I mGluR antagonist, MTEP, was added to the culture during LPS stimulation (Figure 2A-D). In TD controls, cytokine responses tended to be higher with significantly increased production of both IL-1 $\beta(P=0.03)$ and IL-10 $(P=0.04)$ detected (Table 2$)$, as anticipated, largely having the opposite trend as was seen with the group mGluR agonist DHPG. Children with FXS also tended to show increased responses, with elevated levels of IL-1 $\beta(P<0.01)$, IL-6 $(P<0.01)$, and IL-10 $(P<0.01)$. However, the production of GM-CSF was significantly decreased $(P<0.01)$ under these conditions (Table 2). When the relative response to LPS and MTEP to LPS alone was compared between TD controls and children with FXS, GM-CSF $((-16.1 \%(-16.5$ to $8.7 \%)$ versus $-44.3 \%(-54.4$ to $-35.6 \%) ; P=0.02)$ responses were reduced significantly more in children with FXS.

The adaptive immune responses were compared between TD controls and children with FXS by measuring cytokine production after culturing PBMC with a cellular mitogen, PHA, which preferentially activates T-cells. No significance differences were apparent between the two groups (Table 3). Further, the addition of DHPG to the PHA cultures did not alter the cytokine production in TD controls. However, a significant decrease in $\mathrm{T}_{\mathrm{H}} 2$ associated cytokines IL-10 $(P<0.01)$ and IL-13 $(P=0.01)$ was observed in children with FXS versus the TD controls (Table 3). Unlike the innate response, no significant differential response between PHA and DHPG relative to PHA alone was apparent between groups (data not shown). The addition of MTEP to the PHA cultures resulted in a moderate but not significant increase in IL-10 production $(P>0.05)$ in TD controls. In children

Table 1 Cytokine levels in lipopolysaccharide (LPS)-stimulated and LPS plus DHPG-stimulated cell cultures

\begin{tabular}{|c|c|c|c|c|c|c|}
\hline \multirow[b]{2}{*}{ Cytokine } & \multicolumn{3}{|c|}{ TD } & \multicolumn{3}{|c|}{ FXS } \\
\hline & LPS & LPS + DHPG & $P$-value & LPS & LPS + DHPG & $P$-value \\
\hline GM-CSF & 50.4 (27.9 to 58.9$)$ & $39.6(19.5$ to 42.9$)$ & $0.04^{\mathrm{a}}$ & 45.2 (17.7 to 70.7$)$ & 55.3 (20.1 to 107.2$)$ & 0.28 \\
\hline $\mathrm{IL}-1 \beta$ & $1,245.3(957.4$ to $1,855.5)$ & $1,567.6(1,160.2$ to $2,235.0)$ & $0.05^{\mathrm{a}}$ & $1,146.0(607.9$ to $1,748.2)$ & $2,326.9$ (952.6 to $3,313.6)$ & $<0.01^{\mathrm{b}}$ \\
\hline IL-6 & $3,203.9(2,043.8$ to $5,14.0)$ & $2,856.1(1,701.6$ to $4,715.8)$ & 0.33 & $2,900.2(1,792.2$ to $5,110.3)$ & $4,366.9(2,047.0$ to $6,251.1)$ & 0.21 \\
\hline IL-10 & 271.2 (106.3 to 765.6$)$ & $100.0(85.0$ to 515.8$)$ & 0.40 & 286.8 (184.0 to 573.0 ) & 179.3 (76.3 to 342.1) & $0.01^{b}$ \\
\hline IL-12(p40) & 59.2 (36.9 to 72.5 ) & 24.1 (16.3 to 51.2) & $0.01^{\mathrm{a}}$ & 25.0 (6.2 to 77.3$)$ & 46.1 (15.1 to 91.0) & 0.37 \\
\hline TNFa & $838.4(626.3$ to $1,034.4)$ & 570.7 (485.1 to 914.5 ) & $0.03^{\mathrm{a}}$ & 724.2 (413.0 to $1,381.2)$ & $1,010.4(458.1$ to $1,510.4)$ & 0.49 \\
\hline
\end{tabular}

Values reported as median (interquartile range) in $\mathrm{pg} / \mathrm{mL}$. All $P$-values were calculated by Wilcoxon matched-pair signed-rank test. ${ }^{\mathrm{a}} P<0.05$; ${ }^{\mathrm{b}} P<0.01$. DHPG, (S)-3,5-dihydroxyphenylglycine; FXS, fragile X syndrome; TD, typically developing. 

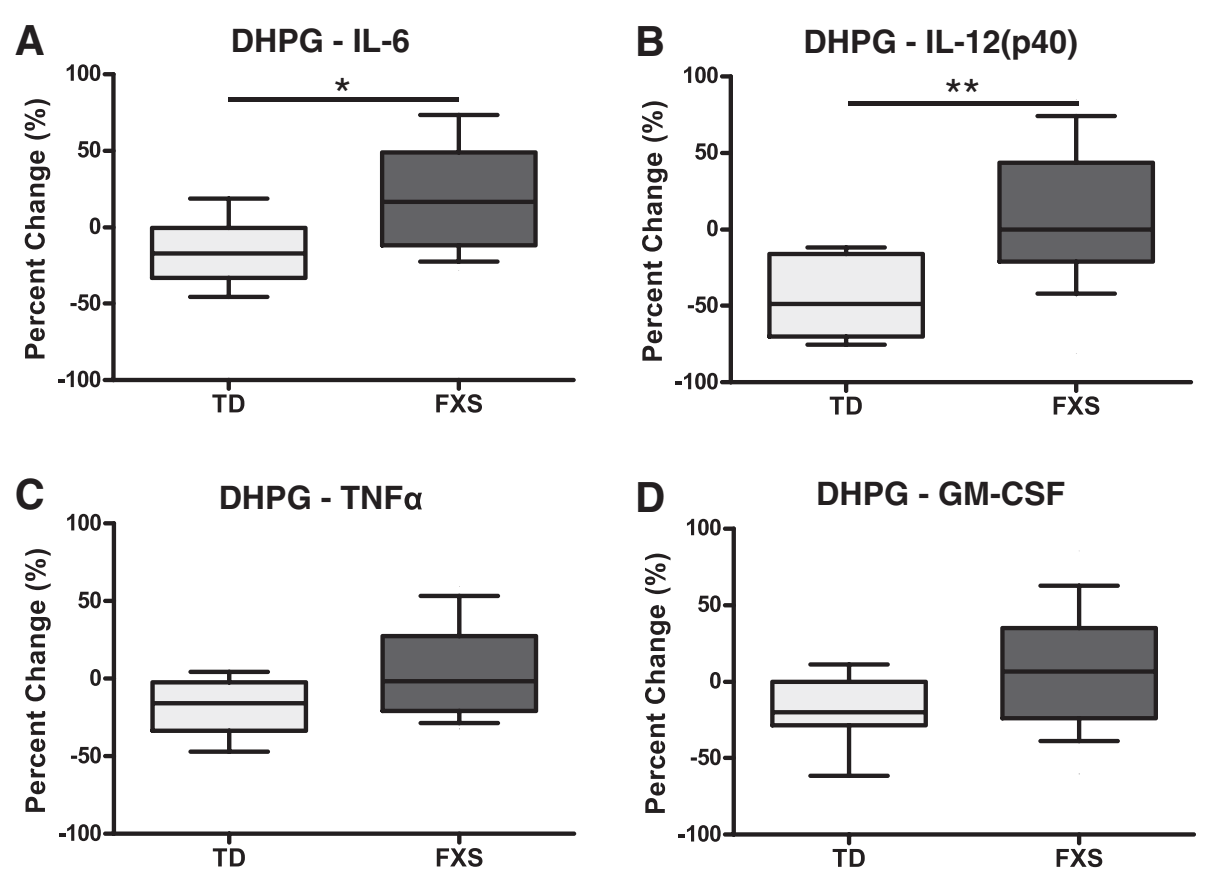

Figure 1 Peripheral blood mononuclear cells' (PBMC) response to lipopolysaccharide (LPS) stimulation with a group I mGluR agonist. PBMC from children with fragile X syndrome (FXS) displayed an opposite or exaggerated immune response to LPS and (S)-3,5-dihydroxyphenylglycine (DHPG) relative to LPS alone when compared with typically developing (TD) controls for (A) IL-6 ( $P=0.02)$, (B) IL-12(p40) $(P<0.01)$, and similar albeit non-significant trends for both (C) TNFa $(P=0.07)$ and (D) GM-CSF $(P=0.11) .{ }^{*} P<0.05$, ${ }^{* *} P<0.01$.
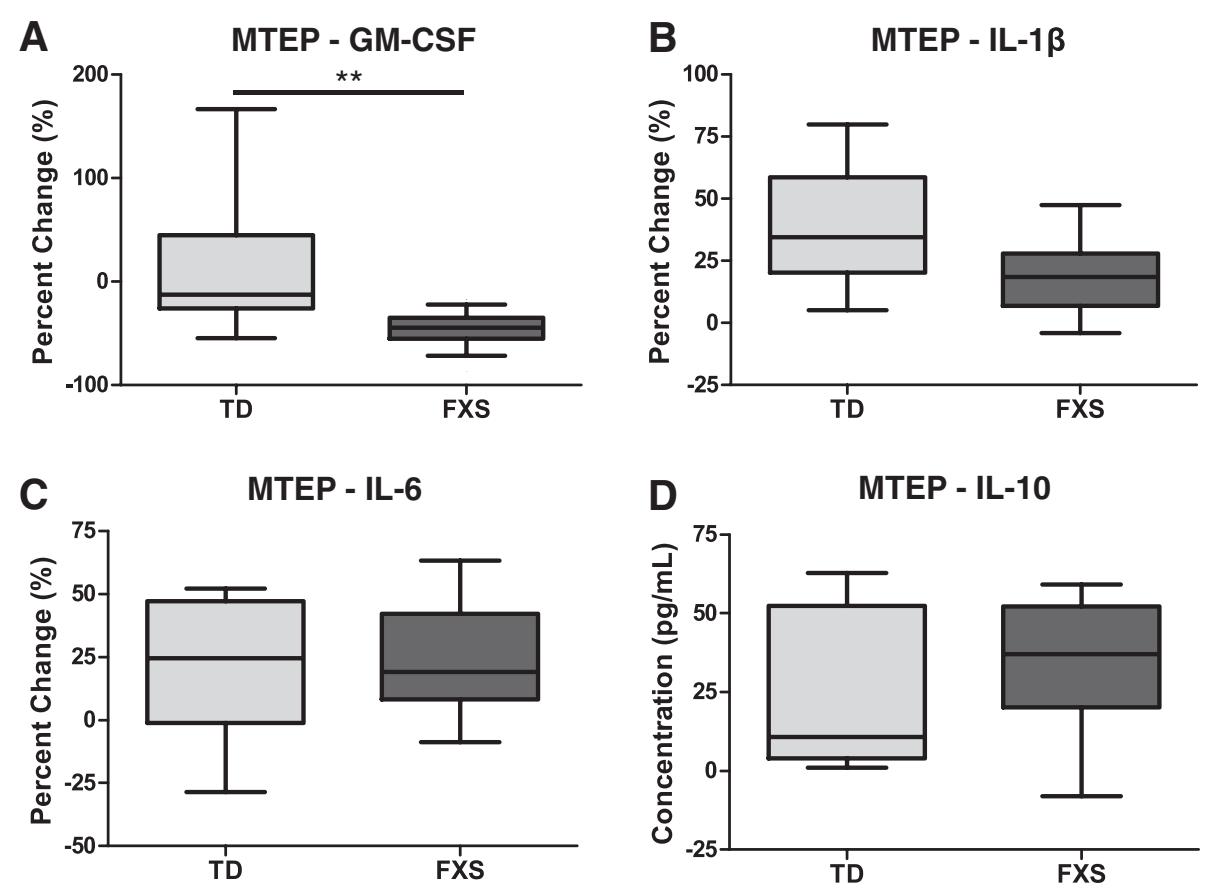

Figure 2 Peripheral blood mononuclear cells' (PBMC) response to lipopolysaccharide (LPS) stimulation with a mGluR5 antagonist. PBMC from children with fragile X syndrome (FXS) displayed an opposite immune response to LPS and 3-((2-methyl-1,3-thiazol-4-yl)ethynyl)pyridine hydrochloride (MTEP) relative to LPS alone when compared with typically developing (TD) controls for (A) GM-CSF $(P<0.01)$. (B) IL-1 $\beta$, (C) IL-6, and (D) IL-10 showed similar responses to MTEP in both subjects and controls. ${ }^{*} P<0.05,{ }^{*} P<0.01$. 
Table 2 Cytokine levels in lipopolysaccharide (LPS)-stimulated and LPS plus MTEP-stimulated cell cultures

\begin{tabular}{|c|c|c|c|c|c|c|}
\hline \multirow[b]{2}{*}{ Cytokine } & \multicolumn{3}{|c|}{ TD } & \multicolumn{3}{|c|}{ FXS } \\
\hline & LPS & LPS + MTEP & $P$-value & LPS & LPS + MTEP & $P$-value \\
\hline GM-CSF & 50.4 (27.9 to 58.9$)$ & 39.8 (36.5 to 49.4$)$ & 0.35 & $45.2(17.7$ to 70.7$)$ & 25.5 (11.7 to 57.6$)$ & $<0.01^{b}$ \\
\hline IL-1 $\beta$ & $1,245.3(957.4$ to $1,855.5)$ & $1,545.9(972.5$ to $2,312.1)$ & $0.03^{\mathrm{a}}$ & $1,146.0(607.9$ to $1,748.2)$ & $1,558.4$ (883.3 to $2,148.6)$ & $<0.01^{\mathrm{b}}$ \\
\hline IL-6 & $3,203.9(2,043.8$ to $4,514.0)$ & $4,195.3(2,235.9$ to $6,071.8)$ & 0.12 & $2,900.2(1,792.2$ to $5,110.3)$ & $3,969.3(2,352.4$ to $7,203.9)$ & $0.01^{\mathrm{a}}$ \\
\hline $\mid \mathrm{IL}-10$ & 271.2 (106.3 to 765.6$)$ & $415.6(168.7$ to $1,076.6)$ & $0.03^{\mathrm{a}}$ & 286.8 (184.0 to 573.0 ) & 389.2 (210.4 to 608.2 ) & $<0.01^{b}$ \\
\hline IL-12(p40) & 59.2 (36.9 to 72.5$)$ & 89.8 (17.3 to 101.8 ) & 0.53 & 25.0 (6.2 to 77.3$)$ & 43.7 (22.5 to 96.3$)$ & 0.44 \\
\hline TNFa & 838.4 (626.3 to $1,034.4)$ & 922.5 (531.7 to $1,190.7)$ & 0.75 & 724.2 (413.0 to $1,381.2)$ & 925.1 (516.7 to $1,557.1)$ & 0.93 \\
\hline
\end{tabular}

Values reported as median (interquartile range) in $\mathrm{pg} / \mathrm{mL}$. All $P$-values were calculated by Wilcoxon matched-pair signed-rank test. ${ }^{\text {a }} P<0.05 ;{ }^{\mathrm{b}} P<0.01$. FXS, fragile $\mathrm{X}$ syndrome; MTEP, 3-((2-methyl-1,3-thiazol-4-yl)ethynyl)pyridine hydrochloride; TD, typically developing.

with FXS, there were decreases in cytokine production with significantly lower levels of GM-CSF $(P<0.01)$, and lower (not significant) levels of IL-13 $(P=0.12)$ (Table 4).

\section{Discussion}

Systemic immune differences have previously been noted in children with FXS compared with TD controls [7]. This included increased IL- $1 \alpha$, as well as decreased RANTES and IP-10 in plasma. In addition, increased rates of infection have been observed in children with FXS, suggesting that immune dysregulation or nonoptimal immune function may occur in these children leading to increased susceptibility to infection [8]. However, it is not clear if this immune dysfunction results from differences in the cellular immune response following immune challenge, or from other physiological factors. In this study we found that peripheral blood immune cells from children with FXS respond to LPS and PHA in a similar fashion as cells from TD children. This suggest that differences in plasma cytokine values previously observed could result from other physiological stresses observed in FXS, particularly increased anxiety, as anxiety levels have been shown to relate to inflammation in a number of studies [25]. Alternatively, immune differences might be restricted to specific anatomical compartments, such as the gastrointestinal tract or liver, which can affect circulating levels of cytokines while analysis of circulating immune cells may not adequately reflect immune differences at these sites.

Group I mGluRs have also been implicated in the pathogenesis of FXS [26]. In FXS, the decreased production of FMRP is thought to lead to a lack of dampening of group I mGluR signaling, resulting in aberrant activation of these receptors. Down-regulation of group I mGluRs in a mouse model of FXS has demonstrated that this is sufficient to ameliorate most of pathological effects seen in FXS [27], suggesting group I mGluRs are highly involved in the pathogenesis of FXS. Although it is difficult, if not impossible, to assess mGluR function in the CNS of children, their peripheral expression (including most immune cells) provides a novel opportunity to assess mGluR function in a non-invasive way. Utilizing this alternative source of mGluRs, we sought to determine if group I mGluR signaling could be assessed in subjects by taking advantage of the known ability of mGluRs to modulate the immune system $[13,28]$. Given the purported mGluR expression and signaling differences in FXS, we hypothesized that analysis of mGluR in the context of immune stimulation could help to explain immune differences previously seen in the periphery in subjects with FXS.

In the presence of the group I mGluR agonist, DHPG, activation of the innate immune system demonstrated that TD controls generally showed a decrease in cytokine

Table 3 Cytokine levels in phytohemagglutinin (PHA)-stimulated and PHA plus DHPG-stimulated cell cultures

\begin{tabular}{|c|c|c|c|c|c|c|}
\hline \multirow[b]{2}{*}{ Cytokine } & \multicolumn{3}{|c|}{ TD } & \multicolumn{3}{|c|}{ FXS } \\
\hline & PHA & PHA + DHPG & $P$-value & PHA & PHA + DHPG & $P$-value \\
\hline GM-CSF & $8.4(2.3$ to 76.9$)$ & $9.6(4.6$ to 45.1$)$ & 0.36 & 11.0 (4.2 to 32.7 ) & 8.2 (5.2 to 25.8$)$ & 0.81 \\
\hline IFNY & 9.3 (5.3 to 122.1 ) & 9.9 (5.1 to 113.0 ) & 0.72 & 8.6 (3.0 to 113.2 ) & 5.6 (1.3 to 221.9) & 0.89 \\
\hline IL-10 & 40.3 (17.7 to 144.3$)$ & 34.2 (25.5 to 117.5$)$ & 1.00 & 44.0 (16.5 to 115.0$)$ & 26.9 (15.3 to 74.6$)$ & $<0.01^{\mathrm{b}}$ \\
\hline IL-13 & 6.2 (3.4 to 135.9 ) & $7.4(3.1$ to 82.4$)$ & 0.09 & $6.1(0.2$ to 22.1$)$ & 2.6 (0.2 to 18.8$)$ & $<0.01^{\mathrm{b}}$ \\
\hline IL-17 & 15.4 (6.5 to 70.1$)$ & 15.4 (6.9 to 40.2 ) & 0.73 & 18.0 (2.7 to 43.6$)$ & 12.1 (5.1 to 34.3$)$ & 0.18 \\
\hline
\end{tabular}

Values reported as median (interquartile range) in $\mathrm{pg} / \mathrm{mL}$. All $P$-values were calculated by Wilcoxon matched-pair signed-rank test. ${ }^{\mathrm{b}} P<0.01$. DHPG, (S)-3,5-dihydroxyphenylglycine; FXS, fragile X syndrome; TD, typically developing. 
Table 4 Cytokine levels in phytohemagglutinin (PHA)-stimulated and PHA plus MTEP-stimulated cell cultures

\begin{tabular}{|c|c|c|c|c|c|c|}
\hline \multirow[b]{2}{*}{ Cytokine } & \multicolumn{3}{|c|}{ TD } & \multicolumn{3}{|c|}{ FXS } \\
\hline & PHA & PHA + MTEP & $P$-value & PHA & PHA + MTEP & $P$-value \\
\hline GM-CSF & $8.4(2.3$ to 76.9$)$ & 3.7 (2.6 to 14.5$)$ & 0.83 & $11.0(4.2$ to 32.7$)$ & $3.5(1.2$ to 12.1$)$ & $0.01^{a}$ \\
\hline IFNY & $9.3(5.3$ to 122.1$)$ & 4.4 (1.4 to 14.4$)$ & 0.83 & 8.6 (3.0 to 113.2$)$ & 1.5 (1.3 to 8.6$)$ & 0.23 \\
\hline $\mathrm{IL}-10$ & 40.3 (17.7 to 144.3$)$ & 34.0 (29.4 to 60.8$)$ & 0.05 & $44.0(16.5$ to 115.0$)$ & 40.4 (9.3 to 67.7$)$ & 0.46 \\
\hline $\mathrm{IL}-13$ & 6.2 (3.4 to 135.9$)$ & 8.1 (2.7 to 29.1) & 0.67 & 6.1 (0.2 to 22.1) & 0.2 (0.2 to 9.6$)$ & 0.12 \\
\hline $\mathrm{IL}-17$ & 15.4 (6.5 to 70.1$)$ & 12.6 (6.5 to 35.6$)$ & 0.25 & 18.0 (2.7 to 43.6 ) & 5.9 (0.9 to 20.2) & 0.69 \\
\hline
\end{tabular}

Values reported as median (interquartile range) in $\mathrm{pg} / \mathrm{mL}$. All $P$-values were calculated by Wilcoxon matched-pair signed-rank test. ${ }^{\mathrm{a}} P<0.05$. FXS, fragile $\mathrm{X}$ syndrome; MTEP, 3-((2-methyl-1,3-thiazol-4-yl)ethynyl)pyridine hydrochloride; TD, typically developing.

production relative to LPS alone. This is similar to the response of microglia following administration of DHPG where a decrease the inflammatory response to LPS has been noted [29]. The activation of mGluR5 has also been shown to reduce microglial associated inflammation and neurotoxicity [30]. Thus, PBMC from TD controls respond to DHPG in a manner similar to central myeloid cells, that is decreased inflammatory cytokine response in the presence of immune challenge. However, immune cells from children with FXS when exposed to DHPG in the presence of LPS did not show the same level of inhibition of inflammation as the TD controls. In the CNS, glutamate serves many purposes, including acting as a messenger between neurons and microglia, and it has also been shown that glutamate signaling in microglia could serve a protective role [31]. Therefore, dysregulation of group I mGluRmediated inhibition in microglia would have potentially devastating effects and lead to negative outcomes such as reduced immune regulation with an increase in neuroinflammation. Agents known to reduce neuroinflammation, such as the tetracycline derivative minocycline, have been shown to rescue many of the impairments seen in the FMR1 knockout (KO) mouse when administered during early development [32]. The observed pattern of immune dysregulation following LPS coupled with DHPG in our study would also explain previous observations in the FMR1 KO mouse. When LPS was given to FMR1 KO mice, similar peripheral immune responses were seen in comparison to wild-type (WT) controls, with the KO mice demonstrating both normal peripheral immune responses and normal microglia responses in culture. However, isolates from the brains of these animals, where glutamate levels would be the highest, showed signs of immune activation and neuroinflammation [33].

The addition of DHPG to PHA-stimulated cell cultures resulted in similar levels of cytokine production between children with FXS and controls. In general, results were much more variable than in the LPS cultures. As PHA preferentially activates T-cells, this might relate to previous findings regarding the ability of mGluRs to regulate T-cell immunity. Not only are mGluR1 and
mGluR5 differentially coupled to separate signaling systems in lymphocytes, their surface expression differs depending on the state of the cell. Immature T-cells show little or no mGluR1 expression unlike mature T-cells, and mGluR5 seems to be constitutively expressed by these cells $[15,18]$. As both the phosphatidylinositide 3-kinase (PI3K) and mitogen-activated protein kinase (MAPK) pathways have been suggested to be dysregulated in FXS $[34,35]$, and as these pathways are involved in T-cell maturation and survival [36], altered mGluR expression may be masking the difference in mGluR function in T-cells.

Blocking of group I mGluR through pharmaceutical means has proven to be beneficial in animal models of FXS. In mouse models of FXS, administration of the group I mGluR antagonists, 2-methyl-6-(phenylethynyl) pyridine hydrochloride (MPEP), has been shown to reverse a number of phenotypes including autogenic seizures and abnormal open field exploration [37], deficits in prepulse inhibition [38], decreased mRNA granule expression [39], excess protein in hippocampal slices [40], and increased density of dendritic filopodia in hippocampal cultures [38]. In our study, administration of MTEP to the immune cell cultures stimulated with LPS resulted in lower production of GM-CSF in children with FXS compared with TD controls. Although MTEP is more specific than MPEP for the mGluRs, it has a short half-life and might not have been fully effective in suppressing all mGluR5 for the duration of the stimulation used herein [41]. Several newer inhibitors are in production and might better serve as agents to test the role mGluR antagonist on dynamic immune response.

Although it is not fully known how mGluRs regulate the immune response, many of the pathways mGluRs signal through are convergent with signaling pathways utilized by immune cell receptors. Immune cells sense LPS though TLR4, a pathogen-associated molecular pattern (PAMP) receptor, which leads to a signaling cascade and the activation of the nuclear factor kappa-light-chainenhancer of activated B cells (NFKB) and the MAPK pathways. The activation of these pathways culminates in the activation of transcription factors, which in turn promote 


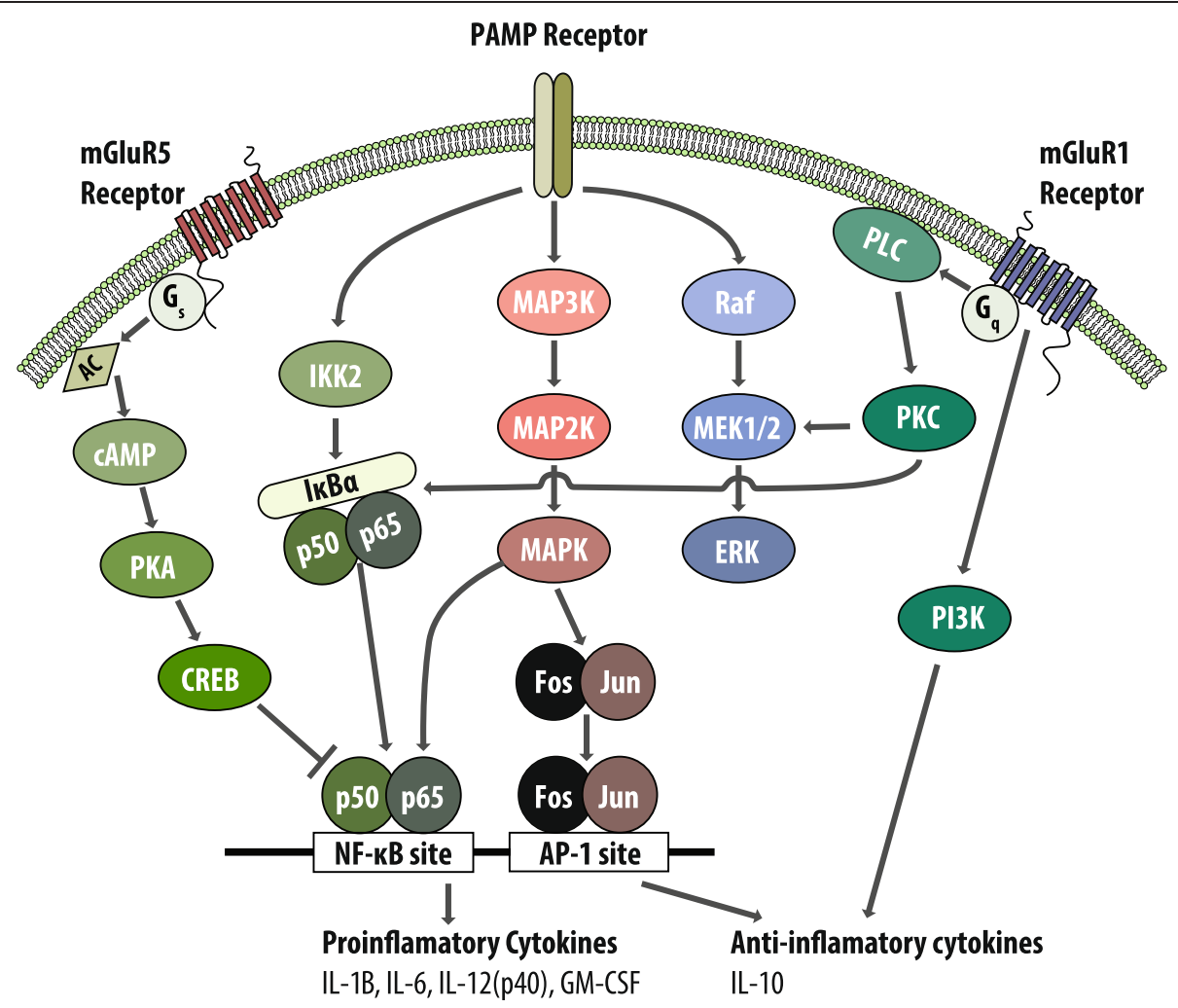

Figure 3 Group I mGluR-signaling pathway in immune cells. Activation of pathogen associated molecular pattern (PAMP) receptors leads to a signaling cascade which can be both inhibited and assisted by group I mGluR signaling.

the production of pro-inflammatory cytokines as well as anti-inflammatory cytokines. Group I mGluRs can inhibit or assist these pathways. Although not fully characterized in myeloid cells, in lymphocytes mGluR1 is coupled to $G_{\alpha q}$ and activates the phospholipase C (PLC), PI3K, and MAPK pathways [18]. mGluR5, however, preferentially couples to $G_{\alpha s}$, which activates the adenylyl cyclase and leads to the production of the secondary messenger 3,5'-cyclic AMP (cAMP) [18]. Whereas mGluR1 pathways tend to assist PAMP signaling, mGluR5 inhibits through the production of cAMP (Figure 3). In FXS, cAMP tends to be lower and cAMP metabolism has been shown to be dysregulated [42-44]. This dysregulation of the cAMP system might be hindering the effects of mGluR5 in myeloid cells in children with FXS, leading to a lack of inhibition of cytokine production when stimulated. This altered response to glutamate by mGluR could have dire effects of the ability of the immune system in the CNS to regulate immune homeostasis, and could have implications into the pathology of FXS.

\section{Conclusion}

Children with FXS did not display an altered dynamic immune response to LPS at a cellular level; however, subjects with FXS showed an altered response to LPS with
DHPG, leading to increased production of inflammatory cytokines. Stimulation of immune cells from subjects with FXS with PHA or PHA with DHPG resulted in no difference in cytokine production in immune cells from similarly treated TD controls. In addition, this study demonstrates that group I mGluR functionality can be tested using a surrogate cellular system instead of neurons. These findings should be valuable in the generation of testable hypothesis to understand how immune and mGluR activation can influence neuronal dysfunction and alter patterns of early brain development in FXS. In addition, mGluR function in PBMC could help to better establish efficacy of drug studies utilizing mGluR-modulating agents.

\section{Abbreviations}

ASD: autism spectrum disorders; CAMP: 3', $5^{\prime}$-cyclic AMP; CNS: central nervous system; DHPG: (S)-3,5-dihydroxyphenylglycine; FBS: fetal bovine serum; FMR1: fragile $X$ mental retardation 1; FMRP: fragile $X$ mental retardation protein; GM-CSF: granulocyte-macrophage colony-stimulating factor; HBBS: Hanks balanced salt solution; IFN: interferon; IL: interleukin; KO: knockout; LOD: limit of detection; LPS: lipopolysaccharides; MAPK: mitogen-activated protein kinase; mGluR: metabotropic glutamate receptor; MPEP: 2-methyl-6-(phenylethynyl) pyridine hydrochloride; MTEP: 3-((2-methyl-1,3-thiazol-4-yl)ethynyl)pyridine hydrochloride; NFkB: nuclear factor kappa beta; PAMP: pathogen-associated molecular pattern; PBMC: peripheral blood mononuclear cells; PCR: polymerase chain reaction; PHA: phytohemagglutinin; PI3K: phosphatidylinositide 3-kinase; PLC: phospholipase C; TCM: tissue culture medium; TD: typically developing; TLR: Toll-like receptor; TNF: tumor necrosis factor; WT: wild-type. 


\section{Competing interests}

$\mathrm{RJH}$ has received funding from Novartis, Roche, Seaside Therapeutics, Curemark and Forest to carry out treatment studies in fragile $X$ syndrome and autism. $\mathrm{RJH}$ is also on the fragile X advisory boards for Novartis and Roche/Genentech regarding treatment studies. FT has received funding from Roche and consult with Novartis and Genentech. The other authors declare that they have no competing interests.

\section{Authors' contributions}

$M C$ and PA designed the study. MC and TN performed the experiments in the study. MC and KB analyzed the data performed statistics. FT provided genotyping data. MC, PA, FT, TN, JVW, RJH and KB all took part in writing the manuscript and critically revised the manuscript. All authors read and approved the final manuscript.

\section{Acknowledgement}

This work was supported by National Institute of Health (NIH) grants HD036071, HD02274, the Jane Botsford Johnson Foundation, Autism Research Institute and Peter Emch Foundation. The above-mentioned funding agencies were not responsible for the design and conduct of the study; collection, management, analysis, and interpretation of the data; and preparation, review, or approval of this manuscript. The authors of this publication declare that they have no conflicting financial interest in relation to the work described.

\section{Author details}

${ }^{1}$ Department of Medical Microbiology and Immunology, and the MIND Institute, UC Davis, 2805, 50th Street, Sacramento, CA 95817, USA. ${ }^{2}$ MIND Institute, UC Davis Medical Center, Sacramento, CA, USA. ${ }^{3}$ Department of Biochemistry and Molecular Medicine, UC Davis, Davis, CA, USA. ${ }^{4}$ Division of Rheumatology, Allergy and Clinical Immunology, UC Davis, Davis, CA, USA. ${ }^{5}$ Department of Pediatrics, University of California at Davis, Davis, CA, USA.

\section{Received: 5 March 2014 Accepted: 27 May 2014}

Published: 19 June 2014

\section{References}

1. Fu YH, Kuhl DPA, Pizzuti A, Pieretti M, Sutcliffe JS, Richards S, Verkerk AJMH, Holden JJA, Fenwick RG, Warren ST, Oostra BA, Nelson DL, Caskey CT: Variation of the Cgg Repeat at the Fragile-X Site Results in Genetic Instability - Resolution of the Sherman Paradox. Cell 1991, 67:1047-1058.

2. Verkerk AJ, Pieretti M, Sutcliffe JS, Fu YH, Kuhl DP, Pizzuti A, Reiner O, Richards S, Victoria MF, Zhang FP, Zhang F, Eussen BE, van Ommen GB, Blonden LA, Riggins GJ, Chastain JL, Kunst CB, Galjaard H, Caskey CT, Nelson DL, Oostra BA, Warren ST: Identification of a gene (FMR-1) containing a CGG repeat coincident with a breakpoint cluster region exhibiting length variation in fragile X syndrome. Cell 1991, 65:905-914.

3. Yu S, Pritchard M, Kremer E, Lynch M, Nancarrow J, Baker E, Holman K, Mulley JC, Warren ST, Schlessinger D, Sutherland GR, Richards Rl: Fragile X genotype characterized by an unstable region of DNA. Science 1991, 252:1179-1181.

4. Snow K, Doud LK, Hagerman R, Pergolizzi RG, Erster SH, Thibodeau SN: Analysis of a CGG sequence at the FMR-1 locus in fragile $X$ families and in the general population. Am J Hum Genet 1993, 53:1217-1228.

5. Harris SW, HessI D, Goodlin-Jones B, Ferranti J, Bacalman S, Barbato I, Tassone F, Hagerman PJ, Herman H, Hagerman RJ: Autism profiles of males with fragile X syndrome. Am J Ment Retard 2008, 113:427-438.

6. Hagerman RJ, Hall DA, Coffey S, Leehey M, Bourgeois J, Gould J, Zhang L, Seritan A, Berry-Kravis E, Olichney J, Miller JW, Fong AL, Carpenter R, Bodine C, Gane LW, Rainin E, Hagerman H, Hagerman PJ: Treatment of fragile $\mathrm{X}$-associated tremor ataxia syndrome (FXTAS) and related neurological problems. Clin Interv Aging 2008, 3:251-262.

7. Ashwood P, Nguyen DV, Hessl D, Hagerman RJ, Tassone F: Plasma cytokine profiles in fragile $\mathrm{X}$ subjects: is there a role for cytokines in the pathogenesis? Brain Behav Immun 2010, 24:898-902.

8. Hagerman RJ, Altshul-Stark D, McBogg P: Recurrent otitis media in the fragile $X$ syndrome. Am J Dis Child 1987, 141:184-187.

9. Bear MF, Huber KM, Warren ST: The mGluR theory of fragile $X$ mental retardation. Trends Neurosci 2004, 27:370-377.

10. Ferraguti F, Shigemoto R: Metabotropic glutamate receptors. Cell Tissue Res 2006, 326:483-504
11. Lüscher C, Huber KM: Group 1 mGluR-dependent synaptic long-term depression: mechanisms and implications for circuitry and disease. Neuron 2010, 65:445-459.

12. Aronica E, Gorter JA, Rozemuller AJ, Yankaya B, Troost D: Activation of metabotropic glutamate receptor 3 enhances interleukin (IL)-1betastimulated release of IL-6 in cultured human astrocytes. Neuroscience 2005, 130:927-933.

13. Boldyrev AA, Carpenter DO, Johnson P: Emerging evidence for a similar role of glutamate receptors in the nervous and immune systems. J Neurochem 2005, 95:913-918.

14. Lombardi G, Miglio G, Dianzani C, Mesturini R, Varsaldi F, Chiocchetti A, Dianzani U, Fantozzi R: Glutamate modulation of human lymphocyte growth: in vitro studies. Biochem Biophys Res Commun 2004, 318:496-502

15. Pacheco R, Gallart T, Lluis C, Franco R: Role of glutamate on T-cell mediated immunity. J Neuroimmunol 2007, 185:9-19.

16. Frank MP, Powers RW: Simple and rapid quantitative high-performance liquid chromatographic analysis of plasma amino acids. J Chromatogr $B$ Analyt Technol Biomed Life Sci 2007, 852:646-649.

17. Clements JD, Lester RA, Tong G, Jahr CE, Westbrook GL: The time course of glutamate in the synaptic cleft. Science 1992, 258:1498-1501.

18. Pacheco R, Ciruela F, Casado V, Mallol J, Gallart T, Lluis C, Franco R: Group I metabotropic glutamate receptors mediate a dual role of glutamate in T cell activation. J Biol Chem 2004, 279:33352-33358.

19. Geurts JJ, Wolswijk G, Bo L, Redeker S, Ramkema M, Troost D, Aronica E: Expression patterns of group III metabotropic glutamate receptors mGluR4 and mGluR8 in multiple sclerosis lesions. J Neuroimmunol 2005, 158:182-190.

20. Wechsler D: Wechsler Intelligence Scale for Children - Fourth Edition (WISC-IV) San Antonio: Psychological Corporation, Harcourt Brace; 2003.

21. Sparrow SCD, Balla D: Vineland Adaptive Behavior Scales, Second Edition (VABS-II): Interview Edition Survey Form. Circle, MN: American Guidance Service; 2005.

22. Rutter MBA, Berument SK, Lord C, Pickles A: Social Communication Questionnaire (SCQ). Western Psychological Services: Los Angeles; 2003.

23. Tassone F, Pan R, Amiri K, Taylor AK, Hagerman PJ: A rapid polymerase chain reaction-based screening method for identification of all expanded alleles of the fragile X (FMR1) gene in newborn and high-risk populations. J Mol Diagn 2008, 10:43-49.

24. Filipovic-Sadic S, Sah S, Chen L, Krosting J, Sekinger E, Zhang W, Hagerman PJ, Stenzel TT, Hadd AG, Latham GJ, Tassone F: A novel FMR1 PCR method for the routine detection of low abundance expanded alleles and full mutations in fragile X syndrome. Clin Chem 2010, 56:399-408.

25. Vogelzangs N, Beekman AT, de Jonge P, Penninx BW: Anxiety disorders and inflammation in a large adult cohort. Trans/ Psychiatry 2013, 3:e249.

26. Dolen G, Bear MF: Role for metabotropic glutamate receptor 5 (mGluR5) in the pathogenesis of fragile X syndrome. J Physiol 2008, 586:1503-1508.

27. Dolen G, Osterweil E, Rao BS, Smith GB, Auerbach BD, Chattarji S, Bear MF: Correction of fragile X syndrome in mice. Neuron 2007, 56:955-962.

28. Volpi C, Fazio F, Fallarino F: Targeting metabotropic glutamate receptors in neuroimmune communication. Neuropharmacology 2012, 63:501-506

29. McMullan SM, Phanavanh B, Li GG, Barger SW: Metabotropic glutamate receptors inhibit microglial glutamate release. ASN Neuro 2012 4:323-330.

30. Byrnes KR, Stoica B, Loane DJ, Riccio A, Davis MI, Faden Al: Metabotropic glutamate receptor 5 activation inhibits microglial associated inflammation and neurotoxicity. Glia 2009, 57:550-560.

31. Eyo UB, Wu L-J: Bidirectional microglia-neuron communication in the healthy brain. Neural Plasticity 2013, 2013:1-10.

32. Bilousova TV, Dansie L, Ngo M, Aye J, Charles JR, Ethell DW, Ethell IM Minocycline promotes dendritic spine maturation and improves behavioural performance in the fragile X mouse model. J Med Genet 2009, 46:94-102.

33. Yuskaitis CJ, Beurel E, Jope RS: Evidence of reactive astrocytes but not peripheral immune system activation in a mouse model of fragile $X$ syndrome. Biochimica et Biophysica Acta (BBA)-Molecular Basis of Disease 2010, 1802:1006-1012

34. Sharma A, Hoeffer CA, Takayasu Y, Miyawaki T, McBride SM, Klann E, Zukin RS: Dysregulation of mTOR signaling in fragile $X$ syndrome. J Neurosci 2010, 30:694-702.

35. Weng N, Weiler IJ, Sumis A, Berry-Kravis E, Greenough WT: Early-phase ERK activation as a biomarker for metabolic status in fragile $X$ syndrome. Am J Med Genet B Neuropsychiatr Genet 2008, 147B:1253-1257. 
36. Juntilla MM, Koretzky GA: Critical roles of the PI3K/Akt signaling pathway in T cell development. Immunol Lett 2008, 116:104-110.

37. Yan QJ, Rammal M, Tranfaglia M, Bauchwitz RP: Suppression of two major fragile $\mathrm{X}$ syndrome mouse model phenotypes by the mGluR5 antagonist MPEP. Neuropharmacology 2005, 49:1053-1066.

38. de Vrij FM, Levenga J, van der Linde HC, Koekkoek SK, De Zeeuw Cl, Nelson DL, Oostra BA, Willemsen R: Rescue of behavioral phenotype and neuronal protrusion morphology in Fmr1 KO mice. Neurobiol Dis 2008, 31:127-132.

39. Aschrafi A, Cunningham BA, Edelman GM, Vanderklish PW: The fragile $X$ mental retardation protein and group I metabotropic glutamate receptors regulate levels of mRNA granules in brain. Proc Natl Acad Sci U S A 2005, 102:2180-2185.

40. Osterweil EK, Krueger DD, Reinhold K, Bear MF: Hypersensitivity to mGluR5 and ERK $1 / 2$ leads to excessive protein synthesis in the hippocampus of a mouse model of fragile $X$ syndrome. J Neurosci 2010, 30:15616-15627.

41. Lindemann L, Jaeschke G, Michalon A, Vieira E, Honer M, Spooren W, Porter R, Hartung T, Kolczewski S, Buttelmann B, Flament C, Diener C, Fischer C, Gatti S, Prinssen EP, Parrott N, Hoffmann G, Wettstein JG: CTEP: a novel, potent, longacting, and orally bioavailable metabotropic glutamate receptor 5 inhibitor. J Pharmacol Exp Ther 2011, 339:474-486.

42. Berry-Kravis E, Huttenlocher PR: Cyclic AMP metabolism in fragile $\mathrm{X}$ syndrome. Ann Neurol 1992, 31:22-26.

43. Berry-Kravis $E$, Ciurlionis R: Overexpression of fragile $X$ gene (FMR-1) transcripts increases cAMP production in neural cells. J Neurosci Res 1998, 51:41-48

44. Berry-Kravis E, Hicar M, Ciurlionis R: Reduced cyclic AMP production in fragile X syndrome: cytogenetic and molecular correlations. Pediatr Res 1995, 38:638-643.

doi:10.1186/1742-2094-11-110

Cite this article as: Careaga et al: Group I metabotropic glutamate receptor mediated dynamic immune dysfunction in children with fragile X syndrome. Journal of Neuroinflammation 2014 11:110.

\section{Submit your next manuscript to BioMed Central and take full advantage of:}

- Convenient online submission

- Thorough peer review

- No space constraints or color figure charges

- Immediate publication on acceptance

- Inclusion in PubMed, CAS, Scopus and Google Scholar

- Research which is freely available for redistribution 